\title{
Comment
}

\section{Science and the Internet: be fruitful and multiply?}

\author{
Sveva Avveduto
}

\begin{abstract}
What role and citizenship has the scientific thought on the web, or rather on the social side of the web? Does it benefit from the debate between peers and with the general public, or else does it only risk to become a monologue? How to deal with the number of instruments the Internet is able to provide in making, discussing and disseminating research? These are some of the questions tackled by the reflections from scholars and experts which were the basis for our debate.
\end{abstract}

The Global Information Technology Report ${ }^{1}$ (Dutta and Mia, 2011) points out that the penetration of ICT (Information Communications Technology) and the growth of social networks as a widespread instrument across varied domains have increasingly marked the past decade at an astounding rate. Their development is leading to new changes the report defines as 'transformation 2.0.'

This transformation is nowadays considered as an intrinsic and shared element of individual behaviours and corporate strategies, government initiatives and services to citizens, which are both the result and the cause to the positive impact of ICT.

In general, ICT gives people a wide range of opportunities to interact with the environment and with other people. This kind of interaction is mediated by the technologies available and their systems of clusters which hare highly open and highly characterised at the same time.

The opportunities offered by the development of Web 2.0 have contributed to multiply occasions and interactions. However, sometimes people are not aware enough of the number of limitations and conditions the Internet implies and forces on them. Too often is the Internet simplistically viewed as a solely democratic expression of participation from the bottom.

The concept of 'social network' has preceded (of a few centuries, I would say) the meaning we attach to it today, but the semantic connection which now binds it to Web 2.0 is direct and inevitable: social network equals Internet.

Too often is this equation improperly used to cover a wide territory that includes blogs, microblogs such as Twitter, the Wiki category, first and foremost Wikipedia, the social news containers having a bookmark function and much more. While a perfect taxonomy is not necessary, it would still be useful to put some order, although it is not the purpose of this paper. Here, we would like to return to the debate that took place during a round table devoted to science in the social networks at the last National Congress on Science Communication. ${ }^{2}$ This commentary touches upon a few subjects that will be further developed, among others, in a specific book to be published soon. ${ }^{3}$

The debate at the Congress and the subsequent analysis aimed at surveying how and how often the opportunities offered by social networks are grasped by and for researchers in order to "communicate science." In a broader sense, are Facebook, Twitter, YouTube, the blogosphere used to communicate science and research? How are they used and by whom? Are they exploited enough and with clear and shared purposes or is their use too patchy and fragmented? What future functions may be envisaged? $\mathrm{Can} /$ must research be communicated through social networks and is there anybody more qualified or suitable to do it: the researcher, the populariser, the journalist? The individual or the institution? Is the process to be harnessed or left alone to evolve spontaneously? These are some of the few questions debated at the round table.

And yet, the starting point was further away and probably the discussion went further beyond. The three essays published here are only samples of the wider analysis to be presented in the above-mentioned book. In any case, they make up an excellent reflection around and within the main common thread: science and the web. The paper by Domenico Laforenza, Maurizio Martinelli, Davide Gualerzi reports in a well-documented contribution many data related to the use of the Internet and outlines the situation of 
the digital divide, also at cultural level. It is agreed that filling this gap is the precondition to any subsequent intervention, either basic or more sophisticated.

The paper by Lella Mazzoli takes us at the heart of the sociological debate which unfolds around the reality and the use of the Internet. It describes crucial aspects of the evolution in the web/users relationship according to different interpretations in terms of communication, culture and policies. Twitter, as an emblematic case of an extremely popular social network, is used to mention both the potential of these tools and the analysis work - not only sociological - still to be carried out.

The contribution by Fabio Fornasari has an unusual viewpoint that differs from the usual analysis of social networks and allows us to enter the circular path of the contents, the arrangement of spaces, images and communication through new instruments and a different outlook.

Finally, can one maintain that the scientific thought on the web, or rather on the social side of the web, plays a role and has a function that do not make it mere exhibition and individual narcissism? Can it be an instrument allowing for a confrontation with the others, peers, experts, or even laymen which can stay on a professional research level? Like many questions concerning the use one makes of (perhaps) neutral instruments, the answers are never so clear and the purpose of finding them lies in constantly getting closer to some conclusion, as one looks for them.

Translated by Massimo Caregnato

\section{Notes and references}

S. Dutta and I. Mia eds. (2011) The Global Information Technology Report 2010-2011, WEF, Geneve.

Convegno Nazionale sulla Comunicazione della Scienza, SISSA Trieste 25-26 November 2010,

http://www.mappetrieste.it/cms/convegno.

3 S. Avveduto (editor), Scienza connessa, including papers by (aside from the authors featured in this Jcom issue), Tullio De Mauro, Derrick de Kerckhove, Sergio Maistrello, Davide Bennato, Nico Pitrelli, Laura Sartori.

\section{Author}

Sveva Avveduto, Director, National Research Council, Institute for Research on Population and Social Policies. Italian delegate OECD Committee for Science and Technology Policy RIHR Group.

E-mail: s.avveduto@irpps.cnr.it.

How TO CITE: S. Avveduto, Science and the Internet: be fruitful and multiply?, Jcom 10(02) (2011) C01 